\title{
Scientific note on interrupted sexual behavior to virgin queens and expression of male courtship-related gene fruitless in a gynandromorph of bumblebee, Bombus ignitus
}

\author{
Koshiro MATSUO $^{1}$, Ryohei KuBO ${ }^{2}$, Tetsuhiko SASAKI ${ }^{2}$, Masato ONo ${ }^{1,2}$, Atsushi UGAJIN ${ }^{1,3}$ \\ ${ }^{1}$ Laboratory of Applied Entomology and Zoology, Graduate School of Agriculture, Tamagawa University, Tokyo, Japan \\ ${ }^{2}$ Honeybee Science Research Center, Tamagawa University, Tokyo, Japan \\ ${ }^{3}$ JT Biohistory Research Hall, 1-1, Murasaki-cho, Takatsuki-shi, Osaka 569-1125, Japan
}

Received 1 July 2017 - Revised 5 January 2018 - Accepted 14 February 2018

\begin{abstract}
Gynandromorphy, which is characterized by the coexistence of male and female tissues in a single individual, is known in insects. Gynandromorphs exhibit diverse levels of defects in sexual behavior. The distribution pattern of both sexes within the nervous systems could be responsible for these differences in behavioral traits; however, most studies have mainly focused on the unique external morphological traits of gynandromorphs, and little attention has been paid to the evaluation of nervous systems, at least in Hymenoptera. In this study, we evaluated a gynandromorphic individual of bumblebee (Bombus ignitus) with a bilaterally dimorphic head and thorax (left side, male; right side, female) and a uniformly masculine gaster. The gynandromorph exhibited abnormal sexual behavior; it normally approached and touched virgin queens but rarely made subsequent copulation attempt. Dissection of the gaster indicated that it possessed a set of male reproductive organs. We analyzed the encephalic and antennal expression patterns of the fruitless (fru ) gene, which shows sex-specific alternative splicing conserved in various insect orders and has been thought to act as the master regulator of sexual behavior in male nervous systems. The gynandromorph showed left-side-biased expression of male-type fru transcripts. We discuss a possible mechanism for the observed unusual interruption of sexual behavior of the gynandromorph by reference to the fru expression pattern and our recent findings on the sexually dimorphic response to female-derived pheromones.
\end{abstract}

gynandromorph / bumblebee / sexual behavior / fruitless

Gynandromorphy, which is characterized by the coexistence of male and female tissues in a single individual, is known in insects (Narita et al. 2010). The gynandromorphic individuals with varying degrees of mosaicism exhibit diverse

Electronic supplementary material The online version of this article (https://doi.org/10.1007/s13592-018-0568-0) contains supplementary material, which is available to authorized users.

Corresponding author: A. Ugajin, atsushi.ugajin@brh.co.jp

Koshiro Matsuo and Ryohei Kubo contributed equally to this work.

Manuscript editor: James Nieh levels of defects in sex-specific behavior (Yoshizawa et al. 2009). Few studies, however, have focused on the nervous systems responsible for the sexual behavior of gynandromorphs, at least in Hymenoptera (Brockmann and Brückner 1999), despite an abundance of literature on gynandromorphic individuals (Hinojosa-Díaz et al. 2012).

We analyzed an adult gynandromorph of the bumblebee (Bombus ignitus), which emerged on January 6 , 2015, from our laboratory colony. The head and thorax exhibited clear dimorphism in the external sexual traits, with male characters observed on the left side and female characters on the right, whereas the gaster exhibited a uniformly 
male-like appearance (Figure 1a-c; Online Resource 1: Figure S1, Table SI).

Ten days after the adult eclosion (the timing of sexual maturation), we examined the mating behavior of the gynandromorph and normal male bumblebees by introducing them individually into an observation cage in which ten sexually mature virgin queens were housed (Online Resource 1: Method details). Normal males smoothly exhibited the typical sequence of mating behavior; i.e., approaching a queen, touching her by antennae (inspection), and mounting her with exposed genitalia (copulation attempt) (van Honk et al. 1978), within $20 \mathrm{~min}$ of introduction (Table I). In contrast, the gynandromorph only approached and inspected the queens, without apparent mounting behavior during the first 20 min observation period. A clear copulation attempt finally occurred almost $15 \mathrm{~min}$ after the start of an additional observation (Table I; Movie, https://doi.org/10.6084/m9. figshare.5758842).

After further 5 days of rearing, we dissected the gynandromorph and verified male genitalia, a pair of testes, vas deferens, and accessory glands in the gaster (Figure 1d, e; Online Resource 1: Figure S2). In contrast, the cephalic labial glands, wherein males produce the scent-marking pheromones to call conspecific new queens (Kubo and Ono 2010), showed clear bilateral dimorphism (Online Resource 1: Figure S3).

The gene fruitless (fru), which encodes a transcription factor located at the bottom of the sexual-differentiation cascade, exhibits the conservation of sex-specific splicing in various insect orders including Hymenoptera (Salvemini et al. 2010). Detailed molecular genetic studies of the fruit fly (Drosophila melanogaster) have revealed that the male-specific Fru isoforms are required to a

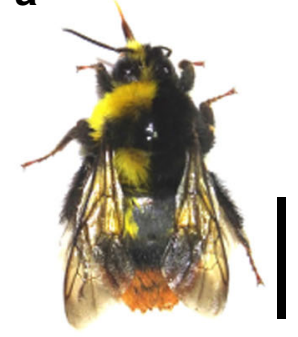

b

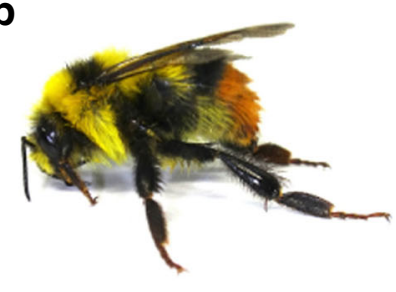

C

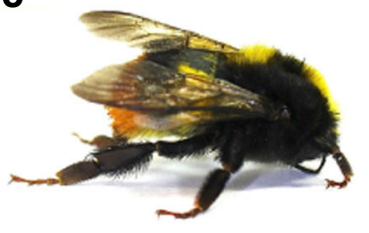

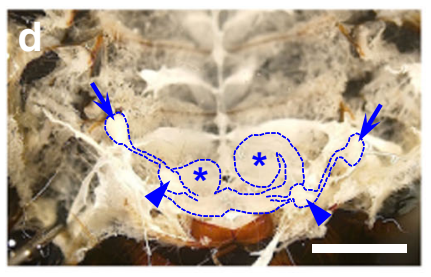
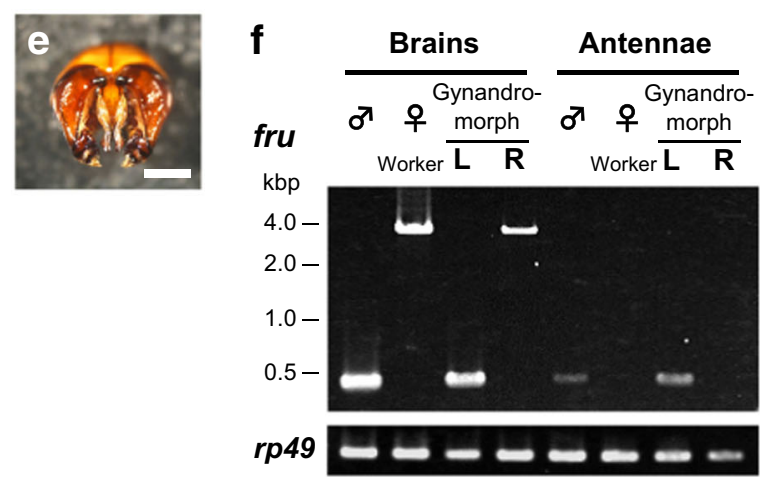

Figure 1. "Quarter female mosaic gynandromorph" of B. ignitus . a-c External appearance of the gynandromorph. Dorsal (a), left-side (b), and right-side (c) views. Bar indicates $1 \mathrm{~cm}$. d Dissection of the gaster from the dorsal aspect. The arrows and arrowheads indicate the pair of testes and vas deferens, respectively. Asterisks indicate the pair of accessory glands. The digestive tract has been removed. Bar indicates $2 \mathrm{~mm}$. e Dissection of the genitalia from the gynandromorph. Bar indicates $1 \mathrm{~mm}$. f Bilaterally dimorphic expression of fru in the brain and antennae. Ribosomal protein 49 ( rp49) was used as positive control of RT-PCR experiments. L, left; R, right. 
Table I. Behavioral response to virgin queens.

\begin{tabular}{llll}
\hline $\begin{array}{l}\text { Individuals (10 days } \\
\text { after adult eclosion) }\end{array}$ & $\begin{array}{l}\text { Relationship with } \\
\text { the gynandromorph }\end{array}$ & $\begin{array}{l}\text { Time elapsed until } \\
\text { copulation attempt }\end{array}$ & $\begin{array}{l}\text { Time elapsed until copulation } \\
\text { attempt in an additional trial }\end{array}$ \\
\hline Male 1 & Unrelated & $4^{\prime} 40^{\prime \prime}$ & \\
Male 2 & Unrelated & $5^{\prime} 43^{\prime \prime}$ & \\
Male 3 & Brother & $6^{\prime} 00^{\prime \prime}$ & \\
Gynandromorph & & $\left(2^{\prime} 40^{\prime \prime}, 3^{\prime} 42^{\prime \prime}, 8^{\prime} 59^{\prime \prime}\right)^{\mathrm{b}}$ & $13^{\prime} 40^{\prime \prime}$ \\
Male 4 & Brother & $13^{\prime} 29^{\prime \prime}$ & \\
Male 5 & Unrelated & $5^{\prime} 38^{\prime \prime}$ & \\
Male 6 & Unrelated & $0^{\prime} 42^{\prime \prime}$ & \\
\hline
\end{tabular}

Behavioral observation was performed in the displayed order. Soon after exhibiting a copulation attempt, males were removed from the cage and replaced with another individual. It took normal males an average of $6^{\prime} 02^{\prime \prime}\left(\mathrm{SD}=4^{\prime} 09^{\prime \prime}\right)$ to exhibit copulation attempt

a "Brother" males and the gynandromorph emerged on the same day from the same colony while "Unrelated" males emerged from different colonies from the gynandromorph

${ }^{\mathrm{b}}$ The gynandromorph exhibited approaching and inspection behavior, but did not attempt copulation during 20 min of observation period. Note that because of the absence of a copulation attempt, the gynandromorph was allowed to stay in the observation cage and exhibited approaching and inspection behavior three times

${ }^{c}$ After the end of all behavioral observation, we again introduced the gynandromorph

establish the courtship-related neural circuits, including sensory, central, and motor systems (reviewed by Yamamoto et al. 2014). Additionally, the knockdown of fru in a more basal insect, the German cockroach (Blattella germanica), resulted in a great reduction of the male sexual behavior. This strongly suggests the widely conserved role of fru as the master regulator of male sexual behavior in insects (Clynen et al. 2011). Using each brain hemisphere divided along the midline and antennae on both sides of the gynandromorph, we performed RT-PCR with the primers which could amplify fru transcripts of $B$. ignitus differing in size between the sexes. The gynandromorph showed bilaterally dimorphic expression pattern of fru: a male-type short product $(<500 \mathrm{bp})$ was selectively amplified from the left-side samples, while from the right side, an approximately 4-kbp amplicon from the brain sample or no clear amplification from the antenna sample was observed to be female-like (Figure 1f; Online Resource 1: Figure S4), implying the leftsided male-type fru-expressing circuit in the gynandromorph.

Visual recognition of a queen is responsible for the approaching behavior by male bumblebees, and an attracted male inspects the cuticle surface of the queen using his antennae. If he detects sex pheromones on the surface of her cuticle, then a copulation attempt could be triggered (van Honk et al. 1978). Therefore, the tendency of the gynandromorph to approach but not attempt copulation could be interpreted as the outcome of the difficulty in the olfactory-dependent transition from early steps of the mating behavior to copulation attempt.

Interestingly, in the fruit fly, even the unilateral activation of the male-type fru-expressing neuron clusters can initiate male-like sexual behavior (Yamamoto et al. 2014). For the bumblebee gynandromorph, one-sided male-type fru-expressing circuit might be sufficient to initiate mating behavior although detailed investigation of the fru expressing neurons in the brain is needed (Online Resource 1: Figure S5).

We recently found that female (both queen and worker) B. ignitus secretes a chemical compound, which exerts a repulsive effect on conspecific females as a contact chemical (R. Kubo, unpublished data). Thus, we assumed that the gynandromorph received opposing information from its dimorphic antennae during the inspection of a queen. The fru-expressing olfactory receptor neurons housed in the left antenna were activated by 
the queens' attractive sex pheromones, whereas the right antenna responded to the repulsive chemical component, resulting in the disturbance of olfactory-dependent transition from approaching to copulation attempt.

Although gynandromorphy occurs at low frequency, detailed analyses would provide us fruitful information regarding the neural mechanisms underlying a variety of sexual behavior on "nonmodel" insects including hymenopteran species.

\section{AUTHORS' CONTRIBUTION}

$\mathrm{KM}$ and $\mathrm{AU}$ conceived this research and designed the experiments; TS and MO participated in the design and interpretation of the data; KM, RK and $\mathrm{AU}$ performed the experiments and analysis; $\mathrm{AU}$ and RK wrote the paper. All authors read and approved the final manuscript.Funding information

This work was supported in part by JSPS KAKENHI Grant Numbers JP14J12036 (Grantin-Aid for JSPS Research Fellow, for AU) and JP26450472 (Grant-in-Aid for Scientific Research (C), for TS).

\section{COMPLIANCE WITH ETHICAL STANDARDS}

Conflict of interest The authors declare that they have no conflict of interest.

Une note scientifique sur le comportement sexuel interrompu chez les reines vierges et l'expression d'un gène fruitless lié à la parade nuptiale d'un bourdon gynandromorphe, Bombus ignitus

gynandromorphe / bourdon / comportement sexuel / fruitless
Eine wissenschaftliche Notiz über das unterbrochene Sexualverhalten gegenüber jungfräulichen Königinnen und Expression des für das männliche Balzverhalten relevanten Gens fruitless bei einem Gynandromorph der Hummel Bombus ignitus

\section{Gynandromorph / Hummel / Sexualverhalten / fruitless}

\section{REFERENCES}

Brockmann, A., Brückner, D. (1999) Dimorphic antennal systems in gynandromorphic honey bees, Apis Mellifera 1. (Hymenoptera: Apidae). Int. J. Insect Morphol. 28, 53-60.

Clynen, E., Ciudad, L., Bellés, X., Piulachs, M.D. (2011) Conservation of fruitless' role as master regulator of male courtship behaviour from cockroaches to flies. Dev. Genes Evol. 221 , 43-48.

Hinojosa-Díaz, I.A., Gonzalez, V.H., Ayala, R., Mérida, J., Sagot, P., Engel, M.S. (2012) New orchid and leafcutter bee gynandromorphs, with an updated review (Hymenoptera, Apoidea). Zoosyst. Evol. 88, 205-214.

Kubo, R., Ono, M. (2010) Comparative analysis of volatile components from labial glands of male Japanese bumblebees (Bombus spp.). Entomol. Sci. 13, 167-173.

Narita, S., Pereira, R.A.S., Kjellberg, F., Kageyama, D. (2010) Gynandromorphs and intersexes- potential to understand the mechanism of sex determination in arthropods. Terr. Arthropod Rev. 3, 63-96.

Salvemini, M., Polito, C., Saccone, G. (2010) fruitless alternative splicing and sex behaviour in insects: an ancient and unforgettable love story? J. Genet. 89, 287-299.

van Honk, C.G.J., Velthuis, H.H.W., Röseler, P.F. (1978) A sex pheromone from the mandibular glands in bumblebee queens. Experientia 34, 838-839.

Yamamoto, D., Sato, K., Koganezawa, M. (2014) Neuroethology of male courtship in Drosophila: from the gene to behavior. J. Comp. Physiol. A 200, 251264.

Yoshizawa, J., Mimori, K., Yamauchi, K., Tsuchida, K. (2009) Sex mosaics in a male dimorphic ant Cardiocondyla kagutsuchi. Naturwissenschaften 96, 49-55. 